\title{
On Extending a Partial Straight-Line Drawing*
}

\author{
Maurizio Patrignani \\ Roma Tre University \\ patrigna@dia.uniroma3.it
}

\begin{abstract}
We investigate the computational complexity of the following problem. Given a planar graph in which some vertices have already been placed in the plane, place the remaining vertices to form a planar straight-line drawing of the whole graph. We show that this extensibility problem, proposed in the 2003 "Selected Open Problems in Graph Drawing" [1, is NP-complete.
\end{abstract}

\section{Introduction}

A (simple) graph $G(V, E)$ consists of a set $V$ of vertices and a set $E$ of vertex pairs called edges. A drawing of $G$ is a mapping of each vertex $v \in V$ to a distinct point of the plane and of each edge $e \in E$ to a Jordan curve connecting its end-vertices. A drawing of $G$ is planar if no pair of edges intersect except, possibly, at common end-vertices. A graph $G$ is planar if it admits a planar drawing. A straight-line drawing of $G$ is a drawing of $G$ where each edge is mapped to a straight segment. Every planar graph admits a straight-line drawing, as independently established by Steinitz and Rademacher [7], Wagner [9], Fary [3], and Stein [6], and such a drawing can be computed in linear time.

In this paper we show that finding a straight-line planar drawing for a graph that is already partially drawn is an NP-complete problem. This extensibility problem was proposed in [1] and thought to be related to the problem of drawing with fixed vertex positions, a problem that was solved by Cabello [2].

Formally, the PARTial DraWing Extensibility problem can be stated as follows.

\section{Problem: Partial Drawing Extensibility (PDE)}

Instance: A planar graph $G(V, E)$ and a mapping between a subset $V^{\prime}$ of its vertices and a set of distinct points of the plane.

Question: Can coordinates be assigned to the vertices in $V-V^{\prime}$ such that the resulting straight-line drawing of $G(V, E)$ is planar?

It can be shown that the PDE problem is in NP. In Section 2 we show that it is also NP-hard. Section 3 concludes the paper.

\footnotetext{
* Work partially supported by European Commission - Fet Open project DELIS Dynamically Evolving Large Scale Information Systems - Contract no 001907, by "Project ALGO-NEXT: Algorithms for the Next Generation Internet and Web: Methodologies, Design, and Experiments", MIUR Programmi di Ricerca Scientifica di Rilevante Interesse Nazionale, and by "The Multichannel Adaptive Information Systems (MAIS) Project", MIUR-FIRB.
} 


\section{NP-Hardness Proof}

In order to show the NP-hardness of the PDE problem we produce a reduction from the Planar 3-Satisfiability (P3SAT) problem, which is strongly NPcomplete [5. P3SAT is defined as follows:

\section{Problem: Planar 3-Satisfiability (P3SAT)}

Instance: A set of clauses $C_{1}, \ldots, C_{m}$ each one having three literals from a set of Boolean variables $v_{1}, \ldots, v_{n}$. A plane bipartite graph $G\left(V_{A}, V_{B}, E\right)$ where nodes in $V_{A}$ correspond to the variables while nodes in $V_{B}$ correspond to the clauses (hence, $\left|V_{A}\right|=n$ and $\left.\left|V_{B}\right|=m\right)$. Edges connect clauses to the variables of the literals they contain. Moreover, $G\left(V_{A}, V_{B}, E\right)$ is drawn without intersections on a rectangular grid of polynomial size in such a way that nodes in $V_{A}$ are arranged in a horizontal line that is not crossed by any edge (see Fig. 11).

Question: Can truth values be assigned to the variables $v_{1}, \ldots, v_{n}$ such that each clause has a true literal?

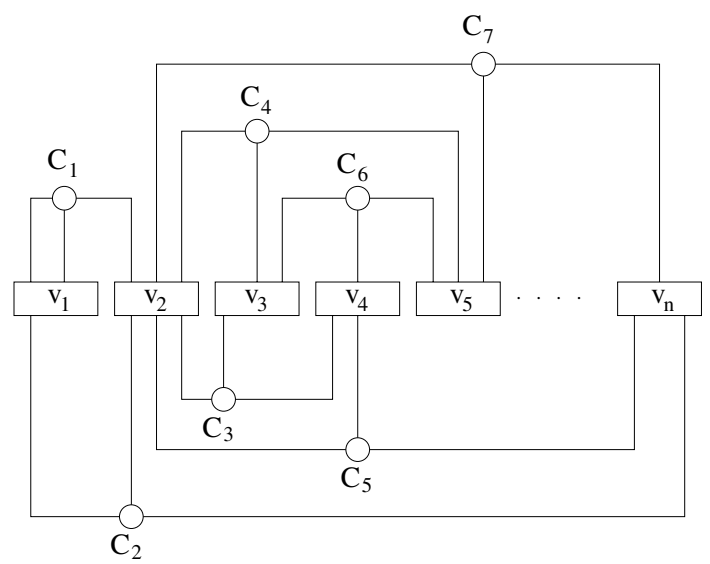

Fig. 1. A planar embedding of graph $G\left(V_{A}, V_{B}, E\right)$ for a P3SAT instance

Given an instance of the PDE problem, we call fixed vertices those in $V^{\prime}$, i.e., those that have assigned coordinates, and we call free vertices those in $V-V^{\prime}$, whose coordinates have to be found in order to obtain a planar straight-line drawing of $G(V, E)$. For the construction of the PDE instance we make use of the basic gadget depicted in Fig. 2. The basic gadget only has fixed vertices, which form the boundary of a chamber. The chamber has two openings on the bottom side, called true gate and false gate, respectively, and labeled with a ' $\mathrm{T}$ ' and an ' $\mathrm{F}$ ' in Fig 2, On the top side the chamber has an even number of openings, that we call exits. The vertices and edges near the exits form narrow corridors pointing towards one of the two gates, and are called true exits or false exits depending on which gate they point to. It can be easily checked from Fig. 2, 


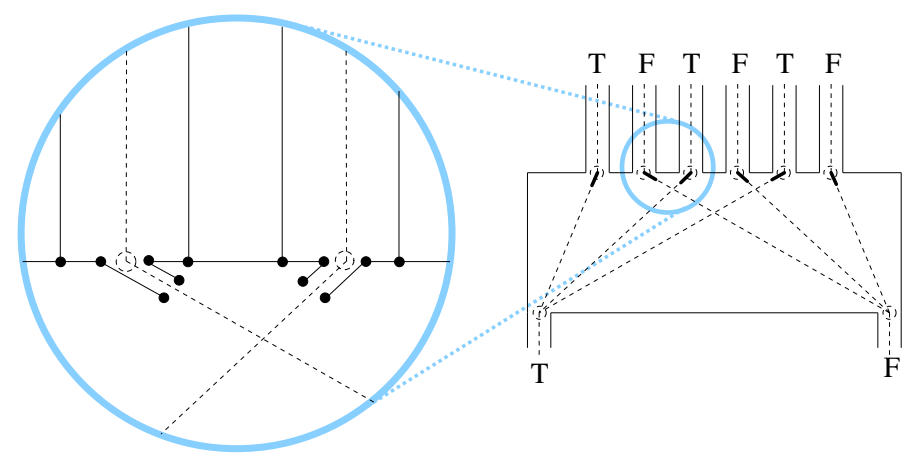

Fig. 2. The basic gadget used to construct the instance of the PDE problem

that only if a path enters a true (resp. false) gate and exits a true (resp. false) exit, it is possible for it to traverse the basic gadget from one gate to one exit leaving only two vertices inside the chamber. In particular, the two vertices of the path must be placed in the spots where dashed circles are drawn in Fig. 2.

Starting from an instance of the P3SAT problem, consisting of the set of clauses $C_{1}, \ldots, C_{m}$, each one having three literals from the Boolean variables $v_{1}, \ldots, v_{n}$, and a drawing of the graph $G\left(V_{A}, V_{B}, E\right)$, an instance of the PDE problem can be constructed as follows. For each variable $v_{i}$ of the P3SAT instance we build a variable gadget depicted in Fig. 3. The variable gadget is composed of two basic gadgets, one of which is mirrored with respect to the horizontal axis. The two basic gadgets are glued together in such a way that their true gates and false gates are attached together. The number of the exits of the top (bottom) basic gadget is equal to two times the number of the edges of $E$ that are incident to the node of $V_{A}$ corresponding to $v_{i}$ from above (below) in the planar drawing of $G\left(V_{A}, V_{B}, E\right)$. Also, the small corridors near the exits point alternatively to the true and to the false gate of each gadget.

Consider a clause $C_{h}=\left(l_{1} \vee l_{2} \vee l_{3}\right)$, where $l_{1}, l_{2}$, and $l_{3}$ are literals of the variables $v_{l_{1}}, v_{l_{2}}$, and $v_{l_{3}}$, respectively. (Variables $v_{l_{1}}, v_{l_{2}}$, and $v_{l_{3}}$ can be assumed to be distinct.) We build a clause gadget corresponding to $C_{h}$ by using three basic gadgets as depicted in Fig. 4. Each basic gadget corresponds to a literal $l_{i}$ and is attached to a true and a false exit of the variable gadget for $v_{l_{i}}$ with two "pipes", called the true and false pipe, respectively, each one bending two times before reaching the variable gadget. Also, the exits of the three basic gadgets point to the same eight points $p_{1}, \ldots, p_{8}$, while, internally, the small corridors near the exits of the chambers point to the true gate or the false gate in such a way that each point $p_{1}, \ldots, p_{8}$ corresponds to a different combination of the truth values of the basic gadget exits. Further, consider the truth assignment for $v_{l_{1}}, v_{l_{2}}$, and $v_{l_{3}}$ that does not satisfy the clause and the point $p_{\text {false }}$ corresponding to it. The corridors pointing to $p_{\text {false }}$ are closed with an edge.

The free vertices of the PDE instance, i.e., those vertices that need to be placed while preserving planarity, are the following. For each variable $v_{i}$, we introduce one free vertex $n_{i, \alpha}$ which is adjacent to the fixed vertex of the variable 


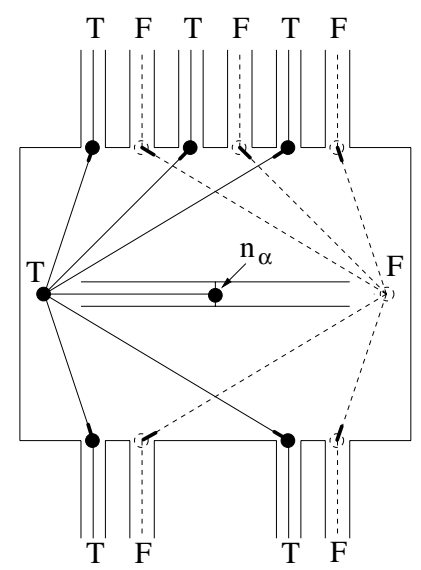

Fig. 3. The variable gadget for a variable which is attached to three clauses from above and to two clauses from below

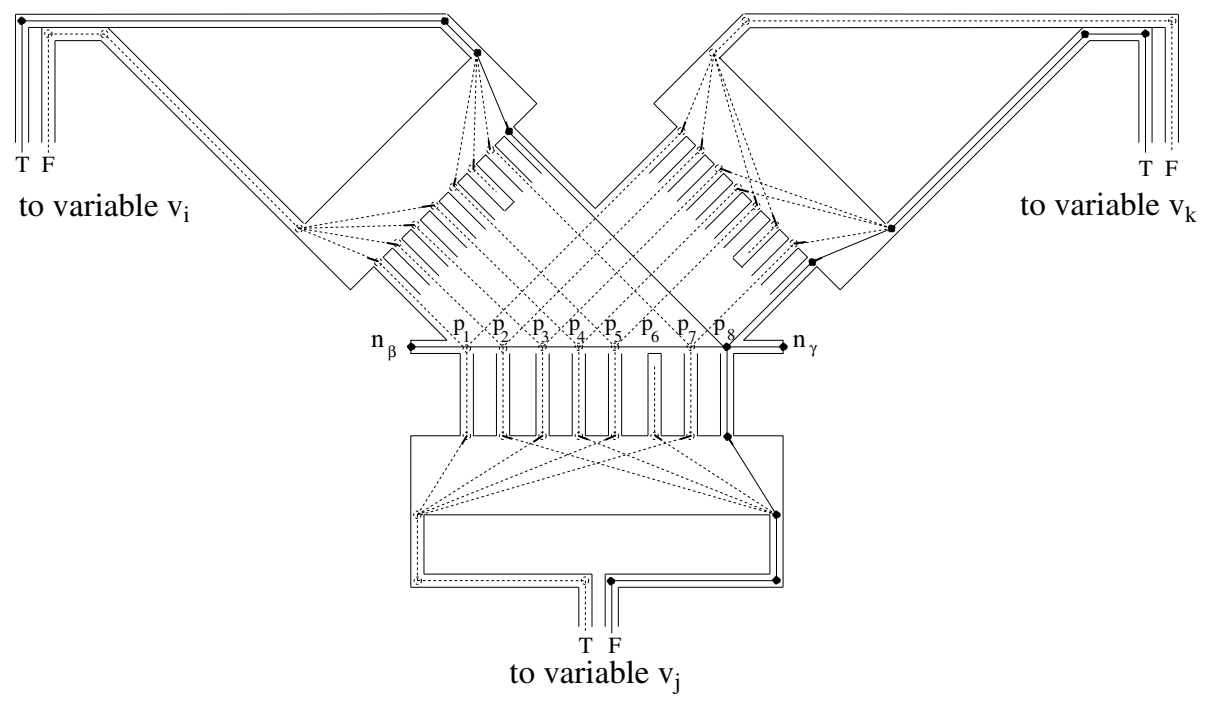

Fig. 4. The clause gadget for a clause $C_{h}=\left(\bar{v}_{i} \vee v_{j} \vee v_{k}\right)$

gadget of $v_{i}$ labeled $n_{\alpha}$ in Fig. 3. For each clause $C_{h}$, we introduce one free vertex $n_{h, \beta, \gamma}$ which is adjacent to the two fixed vertices of the clause gadget corresponding to $C_{h}$ labeled $n_{\beta}$ and $n_{\gamma}$ in Fig. 4. If one literal of variable $v_{i}$ occurs in clause $C_{h}$, vertices $n_{i, \alpha}$ and $n_{h, \beta, \gamma}$ are joined with a path of six edges, that is, containing five other free vertices (see Fig. 50).

Theorem 1. The Partial Drawing Extensibility problem is NP-hard.

Proof. Suppose that the P3SAT instance admits a truth assignment such that each clause has a true literal. A straight-line drawing of the PDE instance can 


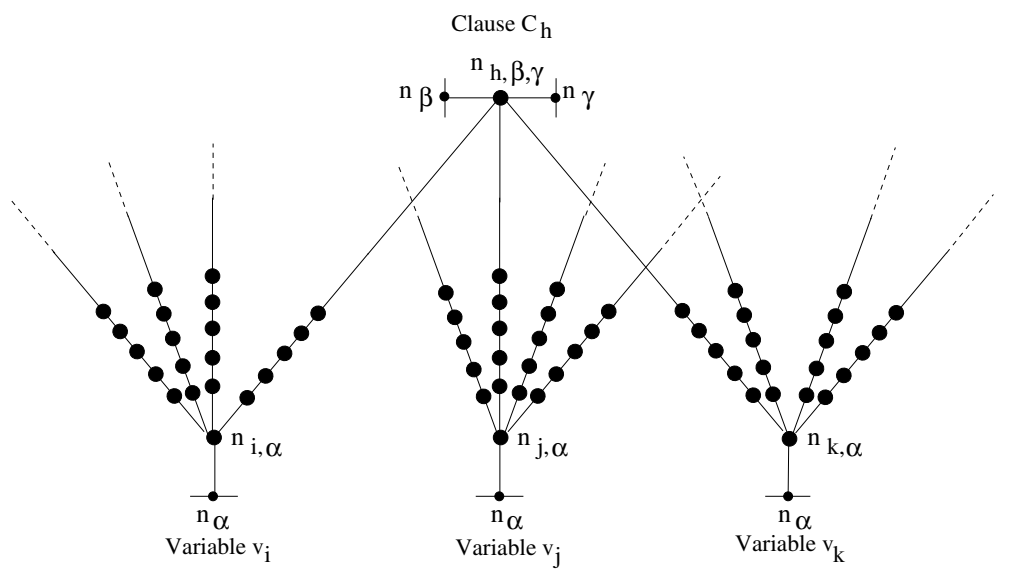

Fig. 5. The free vertices of the PDE instance introduced for a clause $C_{h}$ with literals of the three variables $v_{i}, v_{j}$, and $v_{k}$

be found as follows. Depending on the truth value of variable $v_{i}$ place vertex $n_{i, \alpha}$ of the variable gadget for $v_{i}$ on the true gate or false gate, and let each path to a vertex $n_{h, \beta, \gamma}$ exit from the corresponding true or false exit to clause gadget $C_{h}$. For each clause $C_{h}$ with literals $l_{1}, l_{2}$, and $l_{3}$, place vertex $n_{h, \beta, \gamma}$ on one point $p_{\text {true }}$ different from $p_{\text {false }}$, and let each path to $n_{i, \alpha}$ pass through the (unblocked) corridor of the corresponding basic gadget. The obtained straightline drawing is planar. In fact, edges between fixed vertices do not intersect, and, if $v_{i}$ is true (false), for each clause $C_{h}$ containing a literal of $v_{i}$, the five free vertices between each $n_{i, \alpha}$ and $n_{h, \beta, \gamma}$ can be placed inside the true (false) pipe linking the variable gadget for $v_{i}$ to the clause gadget for $C_{h}$.

Suppose now that the free vertices of the PDE instance can be placed in such a way that the resulting straight-line drawing is planar. A truth assignment for the P3SAT instance such that each clause has a true literal can be easily computed as follows. Assign to each variable $v_{i}$ a true (false) value if the corresponding variable gadget has the vertex $n_{i, \alpha}$ near the true (false) gate. We claim that the truth assignment so computed is such that each clause contains at least a true literal. In fact, consider the clause gadget of clause $C_{h}$. Since the paths attached to $n_{h, \beta, \gamma}$ have five internal vertices only, and since each pipe bends two times, the planarity of the drawing implies that $n_{h, \beta, \gamma}$ is placed on a point $p_{\text {true }}$ different from $p_{\text {false }}$ and that at least one of the three paths joining at $n_{h, \beta, \gamma}$ comes from a variable that has a truth assignment satisfying clause $C_{h}$.

Since, starting from a P3SAT instance, the construction of the corresponding $\mathrm{PDE}$ instance can be done in polynomial time, the statement follows.

\section{Conclusions}

We showed that the Partial Drawing Extensibility problem is NP-complete. For simplicity, in the NP-hardness proof we used a reduction from the P3SAT 
problem producing non-connected PDE instances. We observe that it is not difficult to modify the construction in such a way that the resulting graph is connected. For example, edges can be added to connect each vertex $n_{i, \alpha}$ of the variable gadget for variable $v_{i}$ to the middle point of each horizontal segment of the same variable gadget. Analogous changes performed on clause gadgets will produce a connected graph.

A similar problem to the one addressed in this paper comes up in mesh generation [4, where the already-placed vertices are usually assumed to form a simple polygon and the graph is assumed to have all interior faces triangles. Do these assumptions simplify the problem?

The drawing method of Tutte [8] may be used to show that the problem becomes tractable when the graph is triconnected and the already-placed vertices form convex faces.

\section{Acknowledgments}

We thank the authors of [1] for their help focusing the problem.

\section{References}

1. F. Brandenburg, D. Eppstein, M.T. Goodrich, S. Kobourov, G. Liotta, and P. Mutzel. Selected open problems in graph drawing. In G. Liotta, editor, Graph Drawing (Proc. GD 2003), volume 2912 of Lecture Notes Comput. Sci., pages 515539. Springer-Verlag, 2004.

2. S. Cabello. Planar embeddability of the vertices of a graph using a fixed point set is NP-hard. In Proc. 20th European Workshop on Computational Geometry, 2004.

3. I. Fary. On straight lines representation of planar graphs. Acta Sci. Math. Szeged, 11:229-233, 1948.

4. L.A. Freitag and P.E. Plassman. Local optimization-based untangling algorithms for quadrilateral meshes. In Proc. 10th int. Meshing Roundtable, pages 397-406, 2001.

5. D. Lichtenstein. Planar formulae and their uses. SIAM J. Comput., 11(2):329-343, 1982.

6. S. K. Stein. Convex maps. Proc. Amer. Math. Soc., 2(3):464-466, 1951.

7. E. Steinitz and H. Rademacher. Vorlesungen über die Theorie der Polyeder. Julius Springer, Berlin, 1934.

8. W. T. Tutte. How to draw a graph. Proceedings London Mathematical Society, 13(52):743-768, 1963.

9. K. Wagner. Bemerkungen zum Vierfarbenproblem. Jahresbericht der Deutschen Mathematiker-Vereinigung, 46:26-32, 1936. 